\section{Phasor representation of oxy- and deoxyhemoglobin concentrations: what is the meaning of out-of-phase oscillations as measured by near-infrared spectroscopy?}

\author{
Feng Zheng, Angelo Sassaroli, and Sergio Fantini \\ Tufts University, Department of Biomedical \\ Engineering, 4 Colby Street, Medford, Massachusetts 02155
}

\begin{abstract}
We propose a phasor representation for oscillations at a given frequency (or relatively narrow frequency band) of hemoglobin concentrations in tissue, as well as hemoglobin concentrations measured by near-infrared spectroscopy. This representation provides a straightforward visualization of the phase relationships between oscillations of oxy-, deoxy-, and total hemoglobin concentrations ([HbO], $[\mathrm{Hb}]$, and $[\mathrm{HbT}]$, respectively). We observe that measured oscillations of $[\mathrm{Hb}]$ and $[\mathrm{HbO}]$ with a phase difference that is neither 0 nor $\pi$ must result from the combination of different physiological processes that are out of phase (or time shifted) with respect to each other. Finally, we propose the use of cross-correlation phasors to map phase relationships and correlation levels among hemoglobin oscillations measured at spatially distinct locations at a given frequency. Such a representation may find a natural application in the study of functional connectivity networks in the brain. ( 2010 Society of PhotoOptical Instrumentation Engineers. [DOI: 10.1117/1.3483466]
\end{abstract}

Keywords: near-infrared spectroscopy; diffuse optics; phasors; hemoglobin concentration; functional connectivity.

Paper 10341LR received Jun. 17, 2010; revised manuscript received Jul. 28, 2010; accepted for publication Jul. 28, 2010; published online Aug. 27, 2010.

Near-infrared spectroscopy (NIRS) is a powerful technique for noninvasive studies of biological tissue because of its relatively large penetration depth into tissue (centimeters), and its strong sensitivity to the concentration and oxygenation of hemoglobin. In particular, NIRS allows for measurements of the temporal dynamics of oxyhemoglobin $([\mathrm{HbO}])$ and deoxyhemoglobin $([\mathrm{Hb}])$ concentrations in cerebral tissue, which in human subjects feature natural oscillations at frequencies of $\sim 1 \mathrm{~Hz}$ (arterial pulsation), $\sim 0.2$ to $0.3 \mathrm{~Hz}$ (respiration), $\sim 0.06$ to $0.1 \mathrm{~Hz}$ [low-frequency oscillations (LFOs)] ${ }^{1-3}$ and $\sim 0.04 \mathrm{~Hz}$ [very low frequency oscillations (VLFOs)]. ${ }^{2}$ Functional studies of brain activation that are based on a block protocol (consisting of regular repetitions of activation-rest sequences) also typically investigate hemoglobin concentration changes at the specific frequency of the stimulation

Address all correspondence to: Sergio Fantini. Tel: 617-627-4356; Fax: $617-$ 627-3231; E-mail: sergio.fantini@tufts.edu blocks. ${ }^{2,3}$ Under these common conditions, in which one is interested in hemoglobin concentration oscillations at a specific frequency or over a relatively narrow frequency band, we propose to represent such oscillations using phasor notation. Phasors are polar 2-D vectors characterized by amplitude $(A)$ and phase $(\theta)$, and represent harmonic oscillations at a fixed angular frequency $(\omega)$. In NIRS measurements of tissue hemodynamics, a specific frequency can be isolated by applying a relatively narrow bandpass filter, and the intrinsic phase and amplitude variations can be minimized (and monitored) by considering time windows corresponding to a few periods of oscillations. We define the relative phase of two measured oscillations, say $x_{1}(t)$ and $x_{2}(t)$, at angular frequency $\omega$, in terms of the delay time $\tau_{\max }$, at which the absolute value of the cross-correlation function of the two signals $\left[\left|R_{x 1, x 2}(\tau)\right|\right]$ is maximized. Specifically, the relative phase of $x_{1}(t)$ and $x_{2}(t)$ is defined as $\theta_{x 1, x 2}=\omega \tau_{\max }\left[+\pi\right.$ if $\left.R_{x 1, x 2}\left(\tau_{\max }\right)<0\right]$. We define the phasor amplitude as one half the peak-to-peak range of the measured oscillations, so that the amplitude of the $\vec{x}_{1}$ phasor is given by $\left\{\max \left[x_{1}(t)\right]-\min \left[x_{1}(t)\right]\right\} / 2$.

The concept of phasor representation for the oscillations of $[\mathrm{Hb}]$ and $[\mathrm{HbO}]$ is applied to the model of Fig. 1. In this model, we consider one [Fig. 1(a)] or two [Fig. 1(b)] cubic regions within tissue, where $[\mathrm{HbO}]$ and $[\mathrm{Hb}]$ oscillate at a given frequency $(0.1 \mathrm{~Hz}$ in this example). The hemoglobin oscillations in the time-varying tissue regions are described by the phasors $[\mathrm{HbO}]_{\text {tissue }}$ and $[\overrightarrow{\mathrm{Hb}}]_{\text {tissue }}$ in the respective regions, whereas the hemoglobin oscillations measured at two distinct locations are described by the phasors $[\mathrm{HbO}]_{\text {meas }}$ and $[\overrightarrow{\mathrm{Hb}}]_{\text {meas }}$ at two detector positions. All hemoglobin concentration phasor amplitudes in Fig. 1 are expressed in $\mu \mathrm{M}$. Additional details about the model are provided in the caption of Fig. 1. The measured phasors are obtained by first translating optical intensity changes at two wavelengths, $\Delta I\left(\lambda_{j}\right)(j$ $=1,2)$, into global tissue absorption oscillations $\left[\Delta \mu_{a}^{\left(\lambda_{j}\right)}\right]$ by using the modified Beer-Lambert law ${ }^{4}$ with proper values of the differential path-length factor (DPF) at the two wavelengths considered. Then, the tissue absorption changes are expressed in terms of hemoglobin concentration oscillations by introducing the molar extinction coefficients of oxyhemoglobin and deoxyhemoglobin, $\varepsilon_{\mathrm{HbO}}^{\left(\lambda_{j}\right)}$ and $\varepsilon_{\mathrm{Hb}}^{\left(\lambda_{j}\right)}$, respectively:

$$
\Delta \mu_{a}^{\left(\lambda_{j}\right)}=\varepsilon_{\mathrm{HbO}}^{\left(\lambda_{j}\right)} \Delta[\mathrm{HbO}]_{\text {meas }}+\varepsilon_{\mathrm{Hb}}^{\left(\lambda_{j}\right)} \Delta[\mathrm{Hb}]_{\text {meas }}
$$

The measured global tissue absorption changes can also be expressed in terms of the total mean photon path length $\left(L_{T}\right)$ and the partial mean photon path lengths $\left(l_{i}\right)$ in the $N$ tissue regions that feature hemoglobin oscillations $[N=1$ in Fig. 1(a) and $N=2$ in Fig. 1(b)] as follows:

$$
\Delta \mu_{a}^{\left(\lambda_{j}\right)}=\sum_{i=1}^{N} \frac{\left\langle l_{i}^{\left(\lambda_{j}\right)}\right\rangle}{\left\langle L_{T}^{\left(\lambda_{j}\right)}\right\rangle}\left\{\varepsilon_{\mathrm{HbO}}^{\left(\lambda_{j}\right)} \Delta[\mathrm{HbO}]_{\text {tissue }}^{(i)}+\varepsilon_{\mathrm{Hb}}^{\left(\lambda_{j}\right)} \Delta[\mathrm{Hb}]_{\text {tissue }}^{(i)}\right\}
$$

By combining Eqs. (1) and (2) and solving the resulting linear system of two equations for $j=1$ and $j=2$, one obtains:

1083-3668/2010/15(4)/040512/3/\$25.00 ๑ 2010 SPIE 


$$
\begin{aligned}
\Delta[\mathrm{HbO}]_{\text {meas }}= & \frac{1}{D} \sum_{i=1}^{N}\left\{\varepsilon_{\mathrm{Hb}}^{\left(\lambda_{1}\right)} \varepsilon_{\mathrm{Hb}}^{\left(\lambda_{2}\right)}\left(k_{i}^{\left(\lambda_{1}\right)}-k_{i}^{\left(\lambda_{2}\right)}\right) \Delta[\mathrm{Hb}]_{\text {tissue }}^{(i)}\right. \\
& \left.+\left(\varepsilon_{\mathrm{HbO}}^{\left(\lambda_{1}\right)} \varepsilon_{\mathrm{Hb}}^{\left(\lambda_{2}\right)} k_{i}^{\left(\lambda_{1}\right)}-\varepsilon_{\mathrm{HbO}}^{\left(\lambda_{2}\right)} \varepsilon_{\mathrm{Hb}}^{\left(\lambda_{1}\right)} k_{i}^{\left(\lambda_{2}\right)}\right) \Delta[\mathrm{HbO}]_{\text {tissue }}^{(i)}\right\},
\end{aligned}
$$

$$
\begin{aligned}
\Delta[\mathrm{Hb}]_{\text {meas }}= & \frac{1}{D} \sum_{i=1}^{N}\left\{\left[\varepsilon_{\mathrm{HbO}}^{\left(\lambda_{1}\right)} \varepsilon_{\mathrm{Hb}}^{\left(\lambda_{2}\right)} k_{i}^{\left(\lambda_{2}\right)}-\varepsilon_{\mathrm{HbO}}^{\left(\lambda_{2}\right)} \varepsilon_{\mathrm{Hb}}^{\left(\lambda_{1}\right)} k_{i}^{\left(\lambda_{1}\right)}\right] \Delta[\mathrm{Hb}]_{\text {tissue }}^{(i)}\right. \\
& \left.-\varepsilon_{\mathrm{HbO}}^{\left(\lambda_{1}\right)} \varepsilon_{\mathrm{HbO}}^{\left(\lambda_{2}\right)}\left[k_{i}^{\left(\lambda_{1}\right)}-k_{i}^{\left(\lambda_{2}\right)}\right] \Delta[\mathrm{HbO}]_{\text {tissue }}^{(i)}\right\}
\end{aligned}
$$

where $D=\varepsilon_{\mathrm{HbO}}^{\left(\lambda_{1}\right)} \varepsilon_{\mathrm{Hb}}^{\left(\lambda_{2}\right)}-\varepsilon_{\mathrm{HbO}}^{\left(\lambda_{2}\right)} \varepsilon_{\mathrm{Hb}}^{\left(\lambda_{1}\right)}$, and $k_{i}^{\left(\lambda_{j}\right)}$ are the ratios of partial over total mean path lengths $\left(k_{i}^{\left(\lambda_{j}\right)}=\left\langle l_{i}^{\left(\lambda_{j}\right)}\right\rangle /\left\langle L_{T}^{\left(\lambda_{j}\right)}\right\rangle\right)$. Equations (3) and (4) show that the measured changes in oxyhemoglobin and deoxyhemoglobin concentrations are a linear combination of the hemoglobin concentration changes in the oscillating regions. The linear combinations of $\Delta[\mathrm{Hb}]_{\text {tissue }}^{(i)}$ and $\Delta[\mathrm{HbO}]_{\text {tissue }}^{(i)}$ in Eqs. (3) and (4) correspond to weighted vector sums of the corresponding phasors, according to phasor algebra. This means that if the phase differences between the oscillations of $\Delta[\mathrm{Hb}]_{\mathrm{tissue}}^{(i)}$ and $\Delta[\mathrm{HbO}]_{\text {tissue }}^{(i)}$ are all either 0 or $\pi$, then it is not possible for the phase difference between $\Delta[\mathrm{Hb}]_{\text {meas }}$ and $\Delta[\mathrm{HbO}]_{\text {meas }}$ to assume any value other than 0 (in-phase oscillations) or $\pi$ (opposition-of-phase oscillation). This remains true in general, regardless of tissue heterogeneity, cross talk contributions in Eqs. (3) and (4), or incorrect assumptions for the DPF values at the wavelengths used. This result is important, because physiological hemodynamic or oxygenation changes all induce variations in $\Delta[\mathrm{Hb}]_{\text {tissue }}^{(i)}$ and $\Delta[\mathrm{HbO}]_{\text {tissue }}^{(i)}$ that either have the same sign (0 phase difference) (vascular dilation/constriction, increased/decreased blood volume) or opposite signs ( $\pi$ phase difference) (change in oxygen consumption, speed of blood flow, or oxygenation of incoming blood). ${ }^{3,5}$ It is only a time difference between the onset of such physiological effects (or a phase shift different than 0 or $\pi$ between them) that can account for a phase shift between $\Delta[\mathrm{Hb}]_{\text {meas }}$ and $\Delta[\mathrm{HbO}]_{\text {meas }}$ that is neither 0 nor $\pi .^{5}$ The two (or more) time-delayed or phase-shifted physiological effects can either occur in the same tissue region [Fig. 1(a)] or in different tissue regions [Fig. 1(b)]. In the first case [Fig. 1(a)], there is a non- 0 , non- $\pi$ phase shift between $\Delta[\mathrm{Hb}]_{\text {tissue }}$ and $\Delta[\mathrm{HbO}]_{\text {tissue }}$ in a specific tissue region or vascular compartment. In the second case [Fig. 1(b)], each tissue region (or vascular compartment) features in-phase or opposition-of-phase oscillations of oxyhemoglobin and deoxyhemoglobin concentrations $\left\{\Delta[\mathrm{Hb}]_{\text {tissue }}^{(i)}\right.$ and $\left.\Delta[\mathrm{HbO}]_{\text {tissue }}^{(i)}\right\}$, but there is a non- 0 , non- $\pi$ phase shift between oscillations in different tissue regions. We note that the two cases can be experimentally discriminated, because in the one-region case of Fig. 1(a), the measured phase difference between oxyhemoglobin and deoxyhemoglobin concentrations $\left(\Delta[\mathrm{Hb}]_{\text {meas }}\right.$ and $\left.\Delta[\mathrm{HbO}]_{\text {meas }}\right)$ is independent of the locations of light source and optical detector [if the cross talk term in Eqs. (3) and (4) is negligible], which is not the case of the multipleregion case [two regions in Fig. 1(b)], because the positions of source and detector affect the values of $k_{i}^{\left(\lambda_{j}\right)}$ in Eqs. (3) and (4). We think that considering the interplay of time-shifted physiological processes is the key to interpreting phase shifts of $[\mathrm{Hb}]$ and $[\mathrm{HbO}]$ that are different than 0 or $\pi$, which have

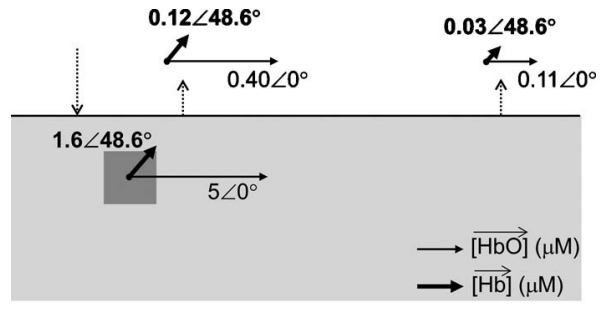

(a)

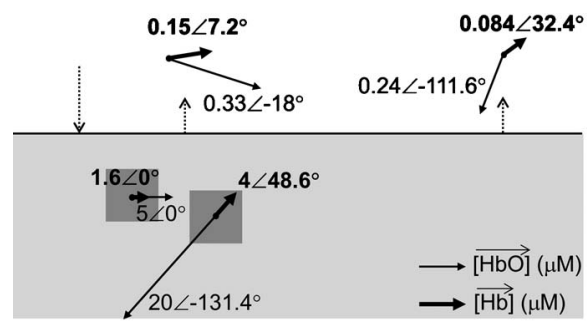

(b)

Fig. 1 Phasor representations of oscillations of deoxyhemoglobin (thick phasors) and oxyhemoglobin (thin phasors) concentrations at localized cubic regions in tissue (dark shaded squares), and as measured by near-infrared spectroscopy with a fixed illumination position (downward dotted arrow) and two collection positions (upward dotted arrows). The frequency of oscillation of the oxyhemoglobin and deoxyhemoglobin concentrations is set at $0.1 \mathrm{~Hz}$. The two illumination-collection distances are 1 and $4 \mathrm{~cm}$. The background tissue (light-shaded area) has a reduced scattering coefficient $\mu_{s}^{\prime}$ $=10 \mathrm{~cm}^{-1}$, and absorption coefficients $\mu_{\mathrm{a}}(690 \mathrm{~nm})=0.12 \mathrm{~cm}^{-1}$ and $\mu_{a}(830 \mathrm{~nm})=0.13 \mathrm{~cm}^{-1}$, corresponding to a total hemoglobin concentration of $60 \mu \mathrm{M}$ and a hemoglobin saturation of $75 \%$. The amplitude and phase of the hemoglobin phasors are indicated in $\mu \mathrm{M}$ and degrees, respectively. (a) Single region of hemoglobin oscillations (cube of $5 \mathrm{~mm}$ side, centered $5 \mathrm{~mm}$ to the right of the illumination point at a depth of $6 \mathrm{~mm}$ ). (b) Two regions of hemoglobin oscillation (second cube of $5 \mathrm{~mm}$ side, centered $13 \mathrm{~mm}$ to the right of the illumination point at a depth of $8 \mathrm{~mm}$ ). In both cases, $[\mathrm{HbO}]_{\text {tissue }}$ and $[\mathrm{Hb}]_{\text {tissue }}$ are represented by the phasors centered at the cubic regions, while $[\mathrm{HbO}]_{\text {meas }}$ and $[\mathrm{Hb}]_{\text {meas }}$ are represented by the phasors at the collection points.

been repeatedly observed and reported in the literature for spontaneous hemoglobin oscillations ${ }^{1,2,6}$ or hemodynamic responses to brain activation. ${ }^{3}$ The word "interplay" in the previous sentence (which has also been used in this context previously ${ }^{1}$ ) can be interpreted here in two conceptually different ways. 1. Two physiological processes within a specific tissue region $i$ actually change their relative timing, thus causing a change in the phase difference between $\Delta[\mathrm{Hb}]_{\text {tissue }}^{(i)}$ and $\Delta[\mathrm{HbO}]_{\mathrm{tissue}}^{(i)}$. 2. A redistribution of background hemoglobin concentration and/or saturation (for example, due to functional brain activation) results in a change of the relative weights in the linear combinations of tissue hemoglobin phasors [Eqs. (3) and (4)], so that even in the absence of any changes in the relative phases of $\Delta[\mathrm{Hb}]_{\text {tissue }}^{(i)}$ and $\Delta[\mathrm{HbO}]_{\text {tissue }}^{(i)}$ (for all values of $i$ ), one would still observe a change in the phase difference between $\Delta[\mathrm{Hb}]_{\text {meas }}$ and $\Delta[\mathrm{HbO}]_{\text {meas }}$.

Figure 2 further illustrates the concept of phasor representation of hemoglobin concentration oscillations by applying it to near-infrared data measured in vivo. The time traces in Fig. 2 represent bandpass filtered $(0.07$ to $0.10 \mathrm{~Hz})$ temporal evolutions of $[\mathrm{Hb}],[\mathrm{HbO}]$, and $[\mathrm{HbT}]$ measured with near- 


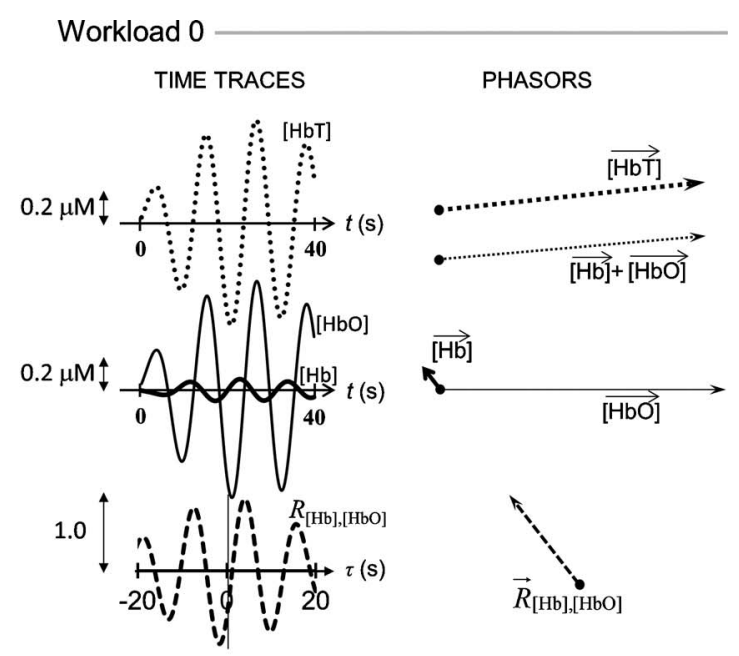

(a)

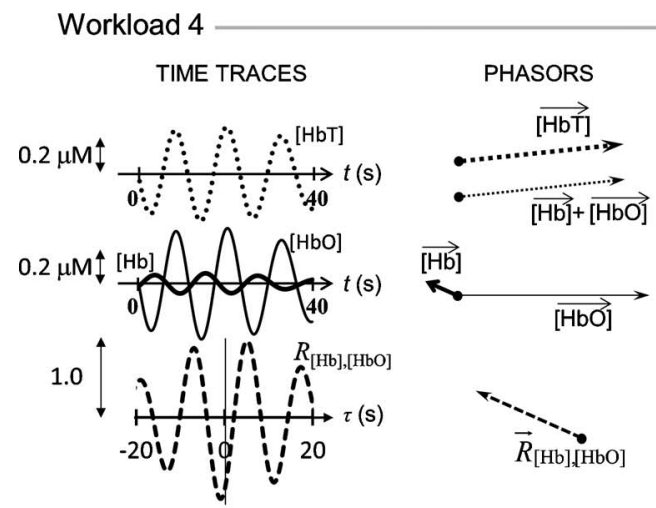

(b)

Fig. 2 Time traces and associated phasors for [HbO], [Hb], [HbT], and for the cross-correlation function $R_{[\mathrm{Hb}],[\mathrm{HbO}]}(\tau)$ measured on the prefrontal cortex of a human subject during (a) a control epoch (Workload 0), and (b) a working-memory-task epoch (Workload 4).

infrared spectroscopy on the prefrontal cortex of a human subject during a previously reported mental workload experiment. ${ }^{7}$ Briefly, the subject was asked to keep track of the number of colored areas in a rotating cube for $40 \mathrm{~s}$. The absence of colored areas is a case referred to as Workload 0 [Fig. 2(a)], whereas the case of four different colors is referred to as Workload 4 [Fig. 2(b)]. The periods of oscillations of the time traces of Fig. 2(a) (11 $\pm 1 \mathrm{~s})$ and Fig. 2(b) $(12 \pm 1 \mathrm{~s})$ are comparable. The variability in the frequency of oscillations is something to monitor because it stretches the applicability of the concept of phasors. The phasor [H $\overrightarrow{b T}]$ defined from the experimental [HbT] trace is typically in excellent agreement with the phasor sum of $[\overrightarrow{\mathrm{Hb}}]$ and $[\mathrm{HbO}]$ (see Fig. 2), confirming the validity of the proposed phasor representation.

Because the cross-correlation function between $[\mathrm{Hb}]$ and $[\mathrm{HbO}]\left[R_{[\mathrm{Hb}],[\mathrm{HbO}]}(\tau)\right]$ is a periodic function (after bandpass filtering of the hemoglobin concentration time traces), it can also be represented as a phasor whose amplitude represents the level of correlation between $[\mathrm{Hb}]$ and $[\mathrm{HbO}]$, and whose phase represents their relative phase difference. We propose to use such cross-correlation phasors to represent the temporal trends of phase relationships and correlation levels between oxyhemoglobin and deoxyhemoglobin oscillations at a specific frequency. Cross-correlation functions $R_{[\mathrm{Hb}],[\mathrm{HbO}]}(\tau)$ measured in the prefrontal cortex during Workloads 0 and 4 are presented in Fig. 2, together with their associated phasors $\vec{R}_{[\mathrm{Hb}],[\mathrm{HbO}]}$. One can see that the level of correlation between $[\mathrm{Hb}]$ and $[\mathrm{HbO}]$, i.e., the amplitude of the cross-correlation phasors, remains about the same under Workload 0 and Workload 4 conditions ( 0.98 and 0.96 , respectively). By contrast, the phase of the $\vec{R}_{[\mathrm{Hb}],[\mathrm{HbO}]}$ phasor, i.e., the phase difference between $[\mathrm{Hb}]$ and $[\mathrm{HbO}]$ oscillations, is greater during Workload 4 (156 deg) than during Workload 0 (128 deg).

Cross-correlation phasors can be used to generate dynamic vectorial maps of hemoglobin concentration oscillations. This can be a powerful tool to characterize networks of functional connectivity in the brain, providing more information than the commonly used correlation coefficient analysis, ${ }^{8}$ which does not take into account and can be negatively impacted by time differences (or phase shifts) between examined signals.

\section{Acknowledgments}

This research was supported by NIH grant R01-NS059933 and by NSF Award IIS-0713506.

\section{References}

1. G. Taga, Y. Konishi, A. Maki, T. Tachibana, M. Fujiwara, and H. Koizumi, "Spontaneous oscillation of oxy- and deoxy-hemoglobin changes with a phase difference throughout the occipital cortex of newborn infants observed using non-invasive optical topography," Neurosci. Lett. 282, 101-104 (2000).

2. H. Obrig, M. Neufang, R. Wenzel, M. Kohl, J. Steinbrink, K. Einhäupl, and A. Villringer, "Spontaneous low frequency oscillations of cerebral hemodynamics and metabolism in human adults," Neuroimage 12, 623-639 (2000).

3. M. Wolf, U. Wolf, V. Toronov, A. Michalos, L. A. Paunescu, J. Choi, and E. Gratton, "Different time evolution of oxyhemoglobin and deoxyhemoglobin concentration changes in the visual and motor cortices during functional stimulation: a near-infrared spectroscopy study," Neuroimage 16, 704-712 (2002).

4. D. T. Delpy, M. Cope, P. van der Zee, S. Arridge, S. Wray, and J. Wyatt, "Estimation of optical pathlength through tissue from direct time of flight measurement," Phys. Med. Biol. 33, 1433-1442 (1988).

5. S. Fantini, "A hemodynamic model for the physiological interpretation of in vivo measurements of the concentration and oxygen saturation of hemoglobin," Phys. Med. Biol. 47, N249-N257 (2002).

6. M. Reinhard, E. Wehrle-Wieland, D. Grabiak, M. Roth, B. Guschlbauer, J. Timmer, C. Weiller, and A. Hetzel, "Oscillatory cerebral hemodynamics-the macro-vs. microvascular level," J. Neurosurg. Sci. 250, 103-109 (2006).

7. A. Sassaroli, F. Zheng, L. H. Hirshfield, A. Girouard, E. T. Solovey, R. J. K. Jacob, and S. Fantini, "Discrimination of mental workload levels in human subjects with functional near-infrared spectroscopy," J. Innov. Opt. Sci. 1, 227-237 (2008).

8. B. R. White, A. Z. Snyder, A. L. Cohen, S. E. Petersen, M. E. Raichle, B. L. Schlaggar, and J. P. Culver, "Resting-state functional connectivity in the human brain revealed with diffuse optical tomography," Neuroimage 47, 148-156 (2009). 